\section{La percepción del colectivo médico sobre la gestión del proceso de desvinculación}

\author{
The medical community's perceptions of \\ management of the layoff process
}

\section{A percepção da comunidade médica sobre a gestão do processo de desvinculação}

Pilar Monreal-Bosch 1

Santiago Perera 1

Maite Martínez González 2

Clara Selva 2

doi: 10.1590/0102-311X00041915

\section{Resumen}

El concepto y el proceso de desvinculación están evolucionando rápidamente. El objetivo del artículo es conocer las percepciones de los profesionales de la medicina de Cataluña (España) sobre el proceso de desvinculación e identificar estrategias y configurar propuestas que permitan un ajuste más satisfactorio. Mediante una aproximación cualitativa, se han realizado 16 entrevistas en profundidad a personas clave del contexto sanitario (fase 1) y 6 grupos focales a 72 personas, atendiendo a criterios muestrales (fase 2). El análisis es interpretacional, siguiendo los supuestos de la Grounded Theory. La comparación y triangulación constante de los resultados generados por las diferentes técnicas e investigadores muestra la representación social que tiene la organización sanitaria actual, inmersa en el nuevo modelo de gestión pública, sobre el colectivo médico y su proceso de desvinculación. En este sentido, la jubilación es vista como un tema personal y ajeno a las políticas de recursos humanos, más interesadas en rejuvenecer plantillas con un "coste" menor. Ante esta situación se proponen alternativas que valoren la pericia y experiencia del médico antes de desvincularse de la organización sanitaria.

Jubilación; Médicos; Investigación Cualitativa

\author{
Correspondencia \\ C. Selva \\ Facultat de Psícologia, Universitat Autònoma de Barcelona. \\ Facultad de Psícologia, Edificio B, Campus Bellaterra, \\ Bellaterra / Barcelona - 08193, España. \\ clara.selva@uab.cat \\ 1 Universitat de Girona, Girona, España. \\ 2 Facultat de Psicología, Universitat Autònoma de Barcelona, \\ Bellaterra, España.
}




\section{Introducción}

En nuestra cultura, el trabajo tiene un significado muy superior a la simple obtención de ingresos económicos. Se convierte en parte del rol central de la edad adulta, que organiza la cotidianidad y proporciona, además de unos ingresos, una actividad significativa como contexto de desarrollo personal, construcción de la identidad y de las relaciones sociales 1 .

Las personas, a lo largo de la carrera profesional, nos enfrentamos a diferentes episodios laborales, caracterizados por cambios de rol y/o cambios de empresa, que configuran nuestra "historia personal". Estos episodios vitales requieren una adaptación de la propia identidad que será más o menos exitosa, y por ende requerirá más o menos desajustes en el propio rol; en función de la situación dada, la propia persona y sus posibilidades de respuesta 2 . En este sentido, es esencial preparar y anticipar estos episodios en aras de favorecer una transición y desvinculación no traumática de la actividad laboral 3,4.

Por el contrario, una decisión forzada de desvinculación en un momento no deseado (es decir, un despido), sería causante de una desvinculación traumática 5,6. En el presente artículo utilizaremos el término "jubilación” para hacer referencia al cese de la actividad laboral; y "desvinculación” para referirnos a la disminución progresiva (entendida como un proceso) de la vinculación psicológica con la actividad laboral 7 .

En el sector sanitario, al cual nos vamos a referir en este artículo, casi un tercio (31\%) de los médicos españoles que trabajan en el Sistema Nacional de Salud tienen entre 50 y 60 años, y un $10 \%$ ha superado ya los 60 años 8 . Estos datos sugieren que una jubilación masiva de profesionales en las próximas décadas puede representar una amenaza para la sostenibilidad del sistema sanitario. En base a estas estadísticas se jubilarán en nuestro país 2.500 médicos al año, llegándose a alcanzar cifras de 7.000 a partir de 2016; cifras superiores a las de nuevos graduados en medicina. Esta realidad pone en el punto de mira a los estudios acerca de la jubilación y el retiro, no sólo referidos al colectivo sanitario, tal como señalan Burke et al. ${ }^{9}$, sino a la población en general. Podría decirse que la jubilación está adquiriendo una atención proporcional al interés que han tenido los estudios sobre desempleo o sobre características organizacionales ligadas al bienestar laboral en los últimos años 7,10,11.

Desde que en 1986 Beehr 12 publicara la primera revisión del fenómeno hasta la actualidad, han sido varias las investigaciones que se han realizado acerca del significado y efectos de la jubilación, recogidas principalmente en handbooks o revisiones 13,14. Las últimas décadas son especialmente significativas, dados los giros que se han planteado en el contexto laboral. Variando desde la edad de la jubilación a las demandas y ofertas que la organización, la sociedad o la propia persona requieren en el momento que se plantea la decisión de desvincularse definitivamente del trabajo.

La investigación sobre la desvinculación laboral se apoya en cuatro enfoques teóricos: (i) la desvinculación como toma de decisión; (ii) la desvinculación como proceso de ajuste; (iii) la desvinculación como etapa del desarrollo profesional; y (iv) la desvinculación como función de los recursos humanos de la organización.

En el primero de estos enfoques se asume que los trabajadores, llegada una edad o previa a ella, toman la decisión de desvincularse de la organización. Como han señalado Wang et al. 15, en esta decisión intervienen factores personales y factores laborales que acontecen en un marco más amplio (social, económico y político). Para Beehr 12, la desvinculación es considerada una toma de decisión progresiva que se va desarrollando a lo largo de un tiempo determinado, siendo un proceso psicológico en el que van a intervenir diferentes variables.

En el segundo enfoque, conocido como proceso de ajuste continuo, las personas pueden decidir desvincularse de su vida profesional en momentos diferentes, habiendo seguido trayectorias distintas, con o sin preparación previa a ese momento y con efectos y cambios totalmente diferentes 16 . Esta perspectiva considera que la transición de la desvinculación en el curso de la vida está relacionada con las historias anteriores de la persona, el contexto de la transición y los caminos que las personas han seguido para llevar a cabo tal transición. En este sentido, algunos favorecedores del ajuste de la persona a la desvinculación serán el contexto laboral más inmediato (e.g., funciones y puestos de trabajo), las redes sociales (e.g., relaciones con los compañeros) y la estructura familiar 17 . Investigaciones recientes en el ámbito sanitario, realizadas bajo este enfoque, señalan tres formas de entender la jubilación que requieren de procesos de ajuste distintos: como una etapa natural a la que enfrentarse, como una posibilidad de cambio para un proyecto vital o como una ruptura vital con repercusiones 
personales, sociales y familiares 6 . Desde este enfoque de ajuste será desde el que se analizarán los resultados del presente artículo.

El tercer enfoque analiza la desvinculación laboral desde una perspectiva más profesional. Este enfoque contrapone la carrera profesional clásica, dirigida por la organización y basada en recompensas, promociones o planes de carrera, con un modelo de carrera dirigido por los propios trabajadores, que tiene en cuenta sus valores y metas personales. Desde este enfoque, la desvinculación se conceptualiza como una etapa tardía del desarrollo de carrera 15 y se reconoce en el trabajador un potencial desarrollado a lo largo de su vida laboral, que permite acciones posteriores diferentes hasta llegar a la edad natural de jubilación. Desde esta perspectiva se habla del empleo-puente (bridge employment) como una salida progresiva de la vida laboral hacia empleos que dan continuidad a su carrera profesional 7,18,19.

Finalmente, desde una perspectiva de los recursos humanos, la desvinculación es analizada como una función que recursos humanos debe llevar a cabo para gestionar, no sólo la ruptura del contrato psicológico de los trabajadores mayores, sino la consecución estratégica de los objetivos de la organización 20. Desde esta visión, el análisis de los contratos psicológicos propuestos desde recurso humanos pone de manifiesto la visión que la organización tiene sobre la desvinculación y el rol de facilitador que asume en su gestión.

Este panorama, y la escasez de estudios en el sector sanitario ${ }^{9}$, nos han llevado a acercarnos a personas que van a enfrentarse a la jubilación o que recientemente se encuentran en este nuevo rol. En este sentido, el objetivo del artículo es conocer las percepciones de los profesionales de la medicina sobre el proceso de desvinculación e identificar estrategias de ajuste más satisfactorias.

\section{Método}

Esta investigación se basa en los supuestos de la perspectiva metodológica cualitativa, donde la realidad que interesa es la que las personas perciben como importante, cómo la experimentan y cómo dan significado a los fenómenos sociales 21. Esta metodología es adecuada para dar respuesta a preguntas de carácter abierto o exploratorio y descubrir relaciones entre variables desconocidas por el investigador 22 .

\section{Participantes}

La selección de la muestra y la recogida de información se han realizado en dos fases que, a pesar de ser consecutivas, en algún momento del tiempo se han desarrollado en paralelo. En el estudio participaron 88 profesionales de la medicina de diferentes zonas de Cataluña, España. De éstos, 16 actuaron como informantes clave en la primera fase (o fase 1) de recogida de datos y 72 integraron los grupos focales realizados en la segunda fase (o fase 2).

En la fase 1, se realizaron 16 entrevistas en profundidad. En donde actuaron como informantes clave 12 hombres y 4 mujeres. De ellos, 4 ocupaban cargos de gerencia, 2 de dirección administrativa, 7 de dirección médica, 1 de gerencia de empresa rural, 1 de presidente del colegio profesional y 1 de médico jubilado.

En la fase 2, se realizaron los grupos focales. Para obtener la máxima variabilidad discursiva se realizó un muestreo teórico intencionado y razonado con los siguientes criterios de inclusión: situación pre- y post-jubilación, sexo, red asistencial (área primaria de salud, hospitalaria, salud mental y sociosanitaria) y contexto donde principalmente han trabajado (rural/urbano). Se realizaron 6 grupos focales, 3 de ellos fueron con participantes prejubilados (1a, 1b, 1c) y los 3 restantes con post-jubilados (2a, $2 \mathrm{~b}, 2 \mathrm{c}$ ), cada uno de los cuales tuvo 12 participantes por grupo. La recogida de información se realizó entre septiembre del 2013 y julio 2014. Consultar Tabla 1 para ver las características de la muestra.

\section{Instrumentos/técnicas}

Las técnicas utilizadas han sido las entrevistas en profundidad en la fase 1 y los grupos focales en la fase 2. Todos los participantes firmaron un consentimiento informado para participar en el estudio.

Las entrevistas en profundidad fueron registradas magnetofónicamente, con una duración aproximada de 60-90 minutos. 
Tabla 1

Características de los participantes por grupos ocupacionales. Cataluña, España, 2013-2014.

\begin{tabular}{|c|c|c|c|c|c|}
\hline \multirow[t]{2}{*}{ Característica } & \multicolumn{2}{|c|}{ Prejubilados (60-65 años) } & \multicolumn{2}{|c|}{ Post-jubilados (66-70 años) } & \multirow[t]{2}{*}{ Total } \\
\hline & Hombres & Mujeres & Hombres & Mujeres & \\
\hline \multicolumn{6}{|l|}{ Red sanitaria } \\
\hline Área primaria de salud & 7 & 8 & 7 & 9 & 31 \\
\hline Hospitalaria & 9 & 3 & 12 & 8 & 32 \\
\hline Salud mental & 2 & 2 & - & - & 4 \\
\hline Socio-sanitaria & 3 & 2 & - & - & 5 \\
\hline Subtotal & 21 & 15 & 19 & 17 & \\
\hline Total & \multicolumn{2}{|c|}{36} & \multicolumn{2}{|c|}{36} & 72 \\
\hline \multicolumn{6}{|l|}{ Contexto de trabajo } \\
\hline Urbano & 15 & 14 & 17 & 11 & 57 \\
\hline Rural & 5 & 2 & 7 & 1 & 15 \\
\hline Subtotal & 20 & 16 & 24 & 12 & \\
\hline Total & \multicolumn{2}{|c|}{36} & \multicolumn{2}{|c|}{36} & 72 \\
\hline
\end{tabular}

Los grupos focales se realizaron con personal sanitario, tuvieron una duración aproximada de 60-90 minutos y fueron registrados en formato digital.

Los temas tratados en ambas fases ( 1 y 2 ) giraban en torno a: cómo ven como profesionales el proceso de desvinculación en su ámbito de trabajo; qué políticas se están implementando en su centro y cómo podría mejorar la organización el proceso de desvinculación de las personas. Los datos obtenidos en ambas fases se transcribieron literalmente con la ayuda del programa informático Soundscriber (http://www-personal.umich.edu/ ebreck/code/sscriber/).

\section{Análisis}

El análisis realizado se basa en los supuestos de la Grounded Theory (Teoría Fundamentada) por el que a partir de los datos textuales se formula la teoría. Este tipo de análisis permite retar el vacío existente entre la teoría y la investigación empírica. Para ello, el investigador, a partir de los relatos de los agentes entrevistados, debe jugar un rol activo en la interpretación de los datos.

Tras un primer pre-análisis, que ha permitido familiarizarnos con el corpus (entendiendo por corpus el total de transcripciones, tanto de las entrevistas, como de los grupos focales) y tener una primera impresión del mismo, el procedimiento analítico se ha dividido en dos etapas diferenciadas y no necesariamente secuenciadas: el nivel textual y el nivel conceptual. En la primera etapa, el nivel textual, se ha fragmentado el corpus en citas y se ha procedido a codificar; es decir, se ha asignado uno o más códigos a cada cita. La estrategia de codificación seguida ha sido bottom-up. Esta estrategia requiere partir de los datos para llegar a conceptos, es decir, la lectura y análisis previo de los datos permite ir elaborando la lista de códigos. En esta estrategia de análisis se segmenta el texto en citas y se crean los códigos con las etiquetas requeridas, o se asignan códigos ya existentes. En el corpus analizado hay un total de 268 citas y 17 códigos.

En la segunda etapa, el nivel conceptual, se han organizado, relacionado y sistematizado las citas y códigos en categorías. En este sentido, las categorías son agrupaciones en base a criterios conceptuales. El proceso de análisis derivó en 3 grandes categorías que subsumen el fenómeno de la jubilación y su representación en los profesionales del ámbito de la medicina, atendiendo a lo que representa para la organización, para la propia persona, y en relación a los valores que se ponen en juego. En el siguiente apartado se describen y conceptualizan pormenorizadamente las categorías y los códigos que las configuran. 
En esta investigación se han llevado a cabo dos tipologías de triangulación: la de los datos (doble recogida de información: entrevistas y grupos focales) y la de los investigadores (tres investigadores han realizado análisis independientes, con un 92\% de acuerdo, incrementando así la calidad y la validez de los datos, al eliminar el sesgo de un único investigador). La triangulación es una buena estrategia de análisis, tanto por el control en el rigor de la investigación, como por la creación de conocimiento para poder realizar propuestas de intervención más adecuadas a las necesidades del colectivo de estudio 23. Para llevar a cabo el análisis se ha utilizado el programa informático de análisis cualitativo Atlas.ti, versión 6.2 (http://atlasti.com/), el cual ha facilitado el proceso de organización y codificación de los datos, y la categorización del texto.

\section{Resultados}

Como se avanzaba, los relatos de los informantes clave y del personal sanitario remiten a tres grandes categorías sobre la jubilación y lo que representa (Tabla 2): lo que representa para la organización (categoría 1), lo que representa para la persona (categoría 2) y la contraposición de valores que supone dentro de la organización (categoría 3).

\section{Tabla 2}

La percepción del colectivo médico sobre la desvinculación. Cataluña, España, 2013-2014.

\begin{tabular}{|c|c|c|}
\hline Categoría & Código & Citas \\
\hline \multirow[t]{4}{*}{$\begin{array}{l}\text { C1. Visión compartida sobre la jubilación: lo } \\
\text { que representa para la organización }\end{array}$} & $\begin{array}{l}\text { La organización está sometida al } \\
\text { cumplimiento de los objetivos económicos }\end{array}$ & $\begin{array}{c}\text { "Por cada dos que jubilamos un residente } \\
\text { cobrará la tercera parte y hará las mismas } \\
\text { visitas o más. Este es el razonamiento de hoy en } \\
\text { día" (GF2b,6) }\end{array}$ \\
\hline & $\begin{array}{l}\text { La experiencia de los profesionales es un } \\
\qquad \text { valor relativo }\end{array}$ & $\begin{array}{c}\text { "Un médico con gran experiencia puede orientar, } \\
\text { decir, opinar, aconsejar (...) Pero ahora no } \\
\text { interesan ni las opiniones ni los consejos. } \\
\text { Interesan los objetivos, los indicadores..." } \\
(\text { GF2c,2) }\end{array}$ \\
\hline & Contraste generacional & $\begin{array}{c}\text { "Los adjuntos, los jóvenes, no les digas tú que se } \\
\text { han de quedar un rato más porque se tiene que } \\
\text { hacer (...) Y no, entonces yo hago el horario, que } \\
\text { es un trabajo muy burocratizado y que a mí no } \\
\text { me gusta nada" (GF2b,10) }\end{array}$ \\
\hline & La desvinculación es un tema personal & $\begin{array}{c}\text { "Llegas a una edad y ya sabes que te toca } \\
\text { jubilarte, nadie te va a solucionar tus problemas, } \\
\text { nadie va a decidir por ti, es una situación } \\
\text { personal, del ámbito privado" (GF2a,10) }\end{array}$ \\
\hline $\begin{array}{l}\text { C2. Visión compartida sobre la jubilación: lo } \\
\text { que representa para la persona }\end{array}$ & $\begin{array}{l}\text { Los profesionales de la medicina siempre } \\
\text { tienen una buena salida }\end{array}$ & $\begin{array}{c}\text { "Somos gente que somos profesionales y que } \\
\text { hemos tenido la suerte de poder hacer funcionar } \\
\text { el cerebro, entonces pienso que tampoco somos } \\
\text { los más pobres del mundo y que podemos ir } \\
\text { tirando" (GF2a,5) }\end{array}$ \\
\hline
\end{tabular}


La categoría 1, que hace referencia a lo que la jubilación representa para la organización, se deriva de 4 códigos:

(1a) La organización está sometida al cumplimiento de los objetivos económicos. Éste es considerado el objetivo principal de la organización. Por lo tanto, ésta puede decidir de forma unilateral y -en función de sus intereses económicos- respecto a la desvinculación de sus profesionales. Es tal el convencimiento de que la misión de la organización es cumplir con los objetivos fijados de atención sanitaria y optimización de recursos que si es necesario ésta utilizará las políticas de desvinculación, como herramienta de regulación (de plantillas, de costes económicos, de funciones, de horarios y/o de salarios de los trabajadores).

Los resultados del estudio reflejan la situación que se está viviendo en el ámbito sanitario, donde la jubilación es tratada desde el plano meramente normativo, sin tener en cuenta a las personas implicadas y al nuevo perfil de persona mayor (más plural y heterogéneo), y prescindiendo también de una vía de diálogo (que correspondería a un modelo de conservación, también contemplado por la ley) que posibilitaría desvinculaciones más tardías y reforzaría envejecimientos más activos 24,25.

(1b) La experiencia de los profesionales es un valor relativo para la organización, pudiendo prescindir de ella cuando resulte cara o poco prioritaria en relación a los objetivos estipulados.

(1c) Contraste generacional. La accesibilidad de la información ha relegado el valor de la experiencia a un lugar secundario. A pesar de que existen evidencias de que los trabajadores mayores tienen un rendimiento en el trabajo equivalente al de los más jóvenes, éstos primeros no resultan tan atractivos y asequibles, económicamente hablando, como los segundos para la organización.

(1d) La desvinculación es un tema personal, no de la organización. La organización sanitaria considera la jubilación como un hecho que marca la desvinculación del trabajador del mundo laboral, poniendo fin a la relación organización-profesional. Así pues, en el ámbito sanitario, la jubilación se ha convertido en una norma a aplicar y su preparación y adaptación forman parte del espacio personal y privado de cada uno de sus trabajadores, donde la organización no siente ninguna responsabilidad.

La categoría 2, que hace referencia a lo que la jubilación representa para la persona, se deriva de dos códigos:

(2a) Los profesionales de la medicina siempre tienen una buena salida. En el ámbito de la práctica médica se comparte la idea de que los médicos son un colectivo que está en muy buena posición para afrontar la jubilación, al igual que cualquier otro reto vital. Dado su nivel cultural, creen que gozan de aficiones socioculturales significativas a las que se pueden dedicar intensamente después de la jubilación, resultando este período muy satisfactorio. Al mismo tiempo, ven una gran oportunidad para la colaboración con organizaciones no gubernamentales y/o programas de voluntariado donde su experiencia profesional sea de utilidad.

(2b) La continuidad de la vida laboral. La mayoría señala que muchos tienen la posibilidad de continuar con la medicina privada y, por lo tanto, esta práctica les permite alargar su vida profesional y acceder a una desvinculación gradual. La práctica privada pasa a perfilarse como un complemento económico y una forma de protegerse y compensar. Esto significa una no-ruptura del ámbito laboral a través de un "trabajo-puente" que presenta efectos muy positivos para el profesional, tal y como se ha señalado en otras investigaciones 7,20,26. Este "trabajo-puente", que no es homogéneo para todo el colectivo, supone un espacio para seguir siendo médico y compensar los efectos de la presión o la ignorancia institucional.

La categoría 3, contrapone valores dentro de la organización, se deriva de 2 códigos:

(3a) Rejuvenecer plantillas versus abaratar costes. Se reconoce la jubilación como una oportunidad para rejuvenecer las plantillas, manteniendo la calidad del servicio y la atención. Aunque se alude también a una forma de abaratar los costes de personal, ya que a los jóvenes se les paga menos y a menudo hacen más horas.

(3b) Pérdida del capital intelectual versus nuevos valores. La jubilación se concibe como la posibilidad de dar entrada al mundo laboral de personal joven y, por ende, de nuevos valores; sin embargo, representa la perdida de otros, como el capital intelectual y humano por el que se ha invertido durante años. Fenómeno conocido como la descapitalización de las personas. En los relatos recogidos hay una constante denuncia a la falta de valor que se está dando a la experiencia, la antigüedad o la pericia de los trabajadores. Se asocia a las personas más veteranas con una imagen estática y pasiva, que para nada corresponde con la visión actual del envejecimiento activo 24. Este hecho 
pone de manifiesto dos cuestiones importantes: (i) las políticas de recursos humanos caen en una evidente discriminación en función de la edad, al ceñirse a la edad normativa para planificar la desvinculación, y (ii) la organización desaprovecha el capital humano de los nuevos perfiles de las personas mayores 27 .

Los relatos de los participantes también aluden a las alternativas que podrían darse para un ajuste más satisfactorio en el proceso de jubilación. Éstas remiten a tres grandes categorías (Tabla 3).

\section{Tabla 3}

Demandas a incorporar en la gestión del proceso de desvinculación. Cataluña, España, 2013-2014.

\begin{tabular}{|c|}
\hline Categoría \\
\hline
\end{tabular}

C1. Incrementar la individualización y la flexibilidad en el proceso de desvinculación

C2. Implicar activamente a las organizaciones en la preparación de la jubilación

C3. Realizar una política activa orientada a los trabajadores más veteranos
Flexibilizar las jubilaciones

Promover y escuchar propuestas de asociaciones y organizaciones profesionales

Buscar y generar recursos en el entorno próximo

Potenciar la atención a las situaciones generadas por los cambios legislativos

Abrir espacios de asesoramiento y participación en las organizaciones

Facilitar el acceso a la preparación de la jubilación

Redefinir funciones y horarios

Diseñar fórmulas para que el conocimientos de los profesionales expertos no se pierda
"Jubilarse poco a poco, según tu situación, es mejor para la persona" $(\mathrm{E}, 5)$

"La administración lo que hace es la norma, escucha, muchas veces hablan de blanco y negro (...) yo creo que si dejasen la iniciativa

a los propios colegios o a las propias instituciones saldrían buenas ideas de aquí. La

administración debería dejar fluir" $(\mathrm{E}, 7)$

"En los entornos rurales la relación de proximidad permite continuar ejerciendo como médico después de la jubilación, ya sea porque los encuentras en tu día a día, en la tienda, en la calle y te preguntan (...) o porque participan en actividades organizadas por el ayuntamiento a nivel colectivo" $(\mathrm{E}, 18)$

"Con tantos cambios algunos compañeros han visto truncadas sus expectativas laborales y sus planes de futuro" (GF1b,2)

"Yo tengo compañeros a los que se les podría prestar ayuda individualizada antes de jubilarse,

porque no saben qué hacer" (GF1a,7)

"Delante de estas dos realidades se ha de intentar hacer planes de preparación, porque en el fondo es una fractura de la vida y es difícil.

Aquí está un poco el reto de trabajar por todo esto" $(\mathrm{E}, 9)$

“La organización debe hacer una política de sensibilización (...) informando con anterioridad

de las alternativas económicas y ofertas empresariales interesantes que, planificadas con tiempo, pueden ayudar a su situación económica en el momento de la desvinculación" $(\mathrm{E}, 11)$

"Reducir las horas de asistencia o formar a los recién llegados me permitiría poder hacer mi trabajo más satisfactoriamente" (GF1b,8)

"Puedo aportar muchas cosas en las comisiones, pues tengo la experiencia de muchos años y se sabe salir de situaciones complejas" (GF2c,9) 
En este caso, la categoría 1, incrementar la individualización y la flexibilidad en el proceso de desvinculación, se deriva de 4 códigos:

(1a) Flexibilizar las jubilaciones. Se alude a la necesidad de un cambio de sistema donde las personas de más de 65 años puedan mantenerse activas en el mundo laboral mediante modalidades flexibles de empleo. Las acciones que los propios agentes ven como necesarias ante la situación descrita y que favorecen el bienestar de las personas mayores, se podrían agrupar en tres categorías. La primera apunta a flexibilizar y personalizar el proceso de desvinculación 28, atendiendo a lo que pueden aportar las organizaciones profesionales, buscando en el contexto más próximo alternativas, y analizando las repercusiones de los cambios legislativos.

(1b) Promover y escuchar las propuestas de asociaciones y organizaciones profesionales, con la finalidad de ampliar las alternativas posibles por parte de la administración y definir así sistemas más dinámicos y particulares a la realidad de los diferentes centros.

(1c) Buscar y generar recursos en el entorno próximo. El entorno próximo se dibuja como una fuente importante de recursos. Éste debe ofrecer actividades (e.g., colaboración en asociaciones o participación en tareas de divulgación de la salud a diferentes colectivos) que generarán un sentimiento de utilidad e identidad personal.

(1d) Potenciar la atención a las situaciones generadas por los cambios legislativos. Los cambios legislativos han generado situaciones complicadas de asumir para los trabajadores, dado que han violentado sus expectativas laborales y sus planes de futuro. En relación a ello, estas situaciones han de ser atendidas por las organizaciones, poniendo a manos de los afectados diferentes servicios y recursos que faciliten la transición.

La categoría 2, implicar activamente a las organizaciones en la preparación a la jubilación, surge a partir de tres códigos:

(2a) Abrir espacios de asesoramiento y participación en las organizaciones. Los profesionales señalan la necesidad de contar con ayudas y espacios de participación dentro de la organización que faciliten la preparación y el proceso de desvinculación. Se relaciona directamente con la participación activa de la organización en la preparación de la jubilación. En este sentido, se señalan acciones como la posibilidad de crear espacios de asesoramiento 29 , facilitar el acceso a la preparación e informar acerca de planes de pensiones.

(2b) Facilitar el acceso a la preparación de la jubilación. Los profesionales y los expertos que han participado en el estudio señalan la necesidad de que las organizaciones faciliten la preparación a la jubilación. Y más especialmente en el caso del colectivo médico que, por definición, suele percibirse a sí mismo como poco necesitado de ayuda. Tal como se ha señalado, y dado que los cambios económicos y sociales son rápidos, no se puede dejar la planificación para el último periodo de la vida laboral, sino que es necesario su planificación mucho más tempranamente y en diferentes fases de la vida.

La categoría 3, realizar una política activa orientada a los trabajadores más veteranos, se deriva de tres códigos:

(3a) Promover planes de pensiones empresariales. Los profesionales de la medicina sugieren que la organización debe hacer una política de sensibilización a los trabajadores activos respecto a la situación económica y profesional post-jubilación.

(3b) Redefinir funciones y horarios. Se sugiere que los trabajadores mayores adopten nuevos roles que les permitan disminuir la carga y funciones habituales en pro de formar a sucesores y transmitir conocimientos y experiencias.

(3c) Diseñar fórmulas para que el conocimiento de los profesionales expertos no se pierda. Las organizaciones deben adaptarse de forma flexible y contextualizada a las nuevas configuraciones de las fuerzas de trabajo, apostando por alargar la vinculación de sus profesionales, complementando sus ingresos y procurando mantener vivo el sentimiento de utilidad y prestigio social de sus profesionales. Las personas mayores que están actualmente en la organización son excelentes asesores o formadores que pueden trabajar con personal más joven (en puestos de trabajo que requieran de la experiencia acumulada en la organización), con el fin de no perder el capital humano ya formado y experto. 


\section{Discusión}

La teoría generada tras la abstracción y significación de los resultados ha aportado diferentes significados en torno al fenómeno de la jubilación para el profesional médico en proceso de desvinculación y para la organización.

En primer lugar, para el profesional médico la jubilación se representa de dos formas: (i) gozando de aficiones socioculturales, nuevas experiencias y retos vitales 6; u (ii) optando por un "trabajopuente" 7,18,19, que permite seguir participando en el mundo laboral. En la primera forma la persona experimenta un nuevo espacio, donde puede disfrutar de otros aspectos de la vida que no sean laborales, mientras que el segundo permite que la persona se desvincule de forma progresiva, atenuando las posibles repercusiones que puede conllevar la jubilación.

En segundo lugar, y para la organización, la jubilación puede utilizarse como herramienta de regulación para alcanzar los objetivos establecidos por la misma 6 . De esta perspectiva se desprende que la persona es un elemento prescindible dentro de la organización y que en realidad no se tiene en cuenta sus potencialidades; dado que no ofrece una vía de diálogo para buscar alternativas que posibiliten desvinculaciones más tardías y envejecimientos más activos 30 . Se suele anteponer los objetivos de la organización a la persona. Esto refleja que no se tiende a dar valor a la experiencia acumulada y al rodaje que presenta la persona más veterana ${ }^{19}$. Además, desde la organización sanitaria se contempla la jubilación como un proceso que tiene que desarrollarse en el espacio personal y privado, donde la organización no presenta ninguna responsabilidad.

Por último, la cultura organizacional actual contrapone valores organizacionales de servicio a valores económicos, por lo que a través de la teoría generada podemos afirmar que las organizaciones sanitarias visualizan el rejuvenecimiento de plantillas como un valor de costes; es decir, sin tener en cuenta la pericia que se pierde y que históricamente ha aportado calidad al servicio. Se enmascara la discriminación por edad en aras de incorporar nuevos valores, tecnológicos, por ejemplo.

\section{Conclusiones}

La gestión activa de las jubilaciones es un tema actual en las organizaciones sanitarias, adquiriendo si cabe mayor importancia desde la aplicación del estatuto marco generado en 2011. Las organizaciones sanitarias, como otras organizaciones de servicios, están actualmente inmersas en procesos de cambios sociales, económicos y políticos. El nuevo modelo de gestión pública, más preocupada por el cumplimiento de objetivos y resultados estratégicos que del valor que aporta la experiencia de la persona, ha impregnado en el funcionamiento de las mismas, de tal manera que la conceptualización del trabajo se ha visto modificada.

Es en este marco que extraemos: (i) la jubilación es un tema personal y no de la organización, es decir, está alejada de las políticas organizacionales; y (ii) la representación social del personal sanitario apoya la primera conclusión señalada: la medicina puede ejercerse siempre, tiene prestigio social y la desvinculación no representa un problema para ellos, ya que el ejercicio privado de la medicina es una alternativa natural a la jubilación.

Entre las propuestas de mejora que los propios usuarios del sistema visualizan para un ajuste más satisfactorio a la nueva situación de jubilación, concluimos con tres estrategias dirigidas al sistema: (i) la necesidad de que la organización personalice las acciones vinculadas a la jubilación; aunque la desvinculación es un proceso individual la organización tiene la responsabilidad de velar por el proceso bien sea con una flexibilidad de la actividad laboral o con la aplicación de las normas estatales vigentes; (ii) más relacionado con las políticas de recursos humanos, la preparación para la jubilación es un proceso que debe realizarse con tiempo (5 últimos años); y (iii) junto a las clásicas propuestas de los planes de pensiones que las organizaciones disponen, se deben diseñar fórmulas que permitan que el conocimiento del profesional no se pierda (sesiones clínicas, comisiones, mentorías, etc.).

A pesar de que los resultados del estudio hacen referencia exclusivamente a un contexto y colectivo determinado, sus aplicaciones prácticas sugieren la necesidad de flexibilizar los procesos de desvinculación, atendiendo a los nuevos contextos socio-laborales y a los nuevos perfiles de persona mayor. Incorporar una perspectiva temporal en los procesos de desvinculación permitirá planificar 
acciones prospectivas a partir de cierta edad, y atender y valorizar el capital humano e intelectual acumulado, es decir, no derrochar el talento.

Estas implicaciones permitirán acercar la organización a las personas y atender y velar por su diversidad. En esta misma línea será interesante, para futuras investigaciones, proponer diferentes roles que las personas jubiladas pueden desarrollar en la organización, como proveedores de experiencias, relaciones y conocimientos, y que resultarían complementarios a lo que aportan las nuevas generaciones (tecnología, idiomas, etc.).

Asimismo, será interesante analizar longitudinalmente si las propuestas efectuadas, una vez que la persona se desvincula de la organización (sea mediante un empleo-puente o no), tienen efecto en su bienestar psicológico. Es decir, analizar los efectos que tiene la experiencia psicológica de envejecer activamente. Al hilo de la limitación principal de este estudio, será necesario recoger las percepciones de otros colectivos profesionales, respecto al proceso de desvinculación, permitiendo conocer desde ámbitos diferentes al de la salud esta realidad y las necesidades que desde éstos se plantean. Por último, será necesario explorar las percepciones del colectivo sanitario (tanto del colectivo sanitario como de otros colectivos a los que se haga extensivo el estudio) desde un diseño de investigación mixto, que permita realizar una triangulación completa de los datos.

\section{Colaboradores}

Todos los autores contribuyeron igualitariamente a la producción del artículo.

\section{Agradecimientos}

Quisiéramos agradecer las inestimables revisiones, comentarios y sugerencias realizados por los revisores respecto al artículo presentado; su alta precisión y acierto han permitido mejorarlo considerablemente. Tanto es así que se han incorporado todos los cambios que sugerían, indistintamente de si se trataban de comentarios o de sugerencias. 


\section{Referencias}

1. Blanch JM. Trabajar y bienestar. Barcelona: Universitat Oberta de Catalunya; 2012.

2. Damman M, Henkens K, Kalmijn M. Missing work after retirement: the role of life histories in the retirement adjustment process. Gerontologist 2013; 2:55-89.

3. Adams G, Rau BL. Putting off tomorrow to do what you want today: planning for retirement. Am Psychol 2011; 66:180-92.

4. von Bonsdorff MB, von Bonsdorff M, Kulmala J, Törmäkangas T, Seitsamo J, Leino-Arjas $\mathrm{P}$, et al. Job strain in the public sector and hospital in-patient care use in old age: a 28-year prospective follow-up. Age Ageing 2014; 43:393-9.

5. Coe N, Zamarro G. Retirement effects on health in Europa. J Health Econ 2011; 30:7786.

6. Martínez M, Monreal-Bosch P. Las desvinculaciones no traumáticas. In: Cantera L, Pallares S, Selva C, editores. Del malestar al bienestar laboral. Barcelona: Editorial Amentia; 2013. p. 181-213.

7. Alcover C, Topa G, Fernández J. La gestión organizacional de los trabajadores mayores y los procesos de mantenimiento, prolongación y salida de la vida laboral. Pap Psicol 2014; 35:91-8.

8. Barber P, González B, Suarez R. Oferta y necesidad de especialistas médicos en España (2010-2025). Madrid: Ministerio de Sanidad y Consumo; 2011. http://www.msc.es/profe sionales/formacion/necesidadEspecialistas/ home.htm.

9. Burke R, Dolan SL, Fiksenbaum L. Predictors of the decision to retire among nurses in Spain. International Journal of Nursing 2013; 1:25-32.

10. Alcover C-M. ¿Ageism en las organizaciones? El papel mediador del apoyo organizacional percibido en las relaciones entre la edad y la ruptura del contrato psicológico. Rev Psicol Organ Trab 2012; 12:299-313.

11. Schalk R, van Veldhoven M, de Lange AH, De Witte H, Kraus K, Stamov-Roßnagel C, et al. Moving European research on work and ageing forward: overview and agenda. European Journal of Work and Organizational Psychology 2011; 19:76-101.

12. Beehr T. The process of retirement: a review and recommendations for future investigation. Pers Psychol 1986; 39:31-55.

13. Sahagún MA, Hermosillo AE, Selva C. La jubilación, hito de la vejez: revisión de aproximaciones psicosociales recientes. Quaderns de Psicologia 2014; 16:27-41.

14. Wang M, Shi J. Psychological research on retirement. Annu Rev Psychol 2014; 65:209-33.
15. Wang M, Adams G, Beehr T, Shultz K. Career issues at the end of one's career: bridge employment and retirement. In: Baugh SG, Sullivan SE, editors. Maintaining focus, energy, and options over the life span. Charlotte: Information Age Publishing; 2009. p. 135-63.

16. van Solinge $H$. Adjustment to retirement. In: Wang M, editor. The Oxford handbook of retirement. New York: Oxford University Press; 2013. p. 311-24.

17. Wang M, Henkens K, van Solinge H. Retirement adjustment: a review of theoretical and empirical advancements. Am Psychol 2011; 66:204-13.

18. Alcover C, Topa G, Parry E, Franccaroli F, Depolo M, editors. Bridge employment: a research handbook. London: Routledge; 2014.

19. Fasbender U, Deller J, Wang M, Wiernik BM. Deciding whether to work after retirement: the role of the psychological experience of aging. J Vocat Behav 2014; 84:215-24.

20. Perera S, Martínez M, Monreal-Bosch P. Managing the process of retirement: the medical professionals' perceptions. Procedia Soc Behav Sci 2013; 82:657-62.

21. Íñiguez L. Investigación y evaluación cualitativa: bases teóricas y conceptuales. Aten Prim 1999; 23:496-502.

22. López J, Blanco F, Scandroglio B, Guttman I. Una aproximación a las prácticas cualitativas en psicología desde una perspectiva integradora. Pap Psicol 2010; 31:131-42.

23. Denzin N. Sociological methods: a sourcebook. Chicago: Aldine Publishing Co.; 1970.

24. López J, Díaz P. La modernización social de la vejez en España. Revista Internacional de Sociología 2013; 71:65-89.

25. Peterson S, Spiker B. Establishing the positive contributory value of older workers: a positive psychology perspective. Organ Dyn 2005; 34:153-67.

26. Dingemans E, Henkens K. Involuntary retirement, bridge employment, and satisfaction with life: a longitudinal investigation. J Organ Behav 2014; 35:575-91.

27. Mariappanadar S. Do retirement anxieties determine bridge employment preference? A study among pre-retirees in the Australian construction industry. Personnel Review 2013; 42:176-204.

28. Spark K, Faragher B, Cooper C. Well-being and occupational health in the 21 st century. J Occup Organ Psychol 2001; 74:489-510.

29. Wöhrmann A, Deller J, Wang M. A mixedmethod approach to post-retirement career planning. J Vocat Behav 2014; 84:307-17.

30. Organización Mundial de la Salud. Envejecimiento activo: un marco político. Rev Esp Geriatr Gerontol 2002; 37:74-105. 


\section{Abstract}

The definition and process of layoff are evolving rapidly. This study focuses on the perceptions of physicians in Catalonia, Spain, concerning layoff and the identification of strategies and proposals that allow more satisfactory adjustment to the process. A qualitative approach was used with 16 in-depth interviews with key persons in the healthcare setting (phase 1) and 6 focus groups with 72 persons, according to sampling criteria (phase 2). The analysis was interpretative, based on Grounded Theory. Comparison and triangulation of the results generated by the different techniques and researchers revealed the social representation of the current healthcare organization (immersed in a new public management model) on the medical community and the layoff process. In this sense, retirement is seen as a personal issue, separate from human resources policies, which are more interested in staff turnover at a lower "cost". Given this situation, the article proposes alternatives that value physicians' experience and expertise before they leave the healthcare organization.

Retirement; Physicians; Qualitative Research

\section{Resumo}

O conceito e processo de desvinculação estão evoluindo rapidamente. O objetivo do trabalho é conhecer as percepções de profissionais médicos na Catalunha (Espanha) sobre o processo de desvinculação e identificar estratégias e propostas de criação de um ajuste mais satisfatório. Através de uma abordagem qualitativa, houve 16 entrevistas em profundidade com pessoas-chave do contexto de saúde (fase 1) e 6 grupos focais com 72 pessoas, com base em critérios de amostragem (fase 2). A análise é interpretativa, seguindo os pressupostos da Grounded Theory. A comparação constante e triangulação dos resultados gerados por diferentes técnicas e pesquisadores mostram a representação social da atual organização da saúde, imersa no novo modelo de gestão, na comunidade médica e o processo de desvinculação. Nesse sentido, a aposentadoria é vista como uma questão pessoal, separada das políticas de recursos humanos, que estão mais interessadas em modelos de rejuvenescimento de pessoal com um "custo" menor. Diante desta situação, o artigo propõe alternativas que valorizam a experiência e os conhecimentos dos médicos antes da desvinculação à organização de saúde.

Aposentadoria; Médicos; Pesquisa Qualitativa
Recibido el 17/Mar/2015

Versión final presentada el 15/Sep/2016

Aprobado el 25/Oct/2016 